\title{
Design and Development of ZigBee Based Instantaneous Flat-plate Collector Efficiency Measurement System
}

\author{
K.Vairamani, K. Arun Venkatesh and N. Mathivanan. \\ University Science Instrumentation Centre, Madurai Kamaraj University, Madurai - 625 021, India. \\ e-mail: vairamani_electro@yahoo.com, arunvenkateshk@gmail.com,nmvanan@yahoo.com.
}

\begin{abstract}
Computing the efficiency of flat-plate collector is vital in solar thermal system testing. This paper presents the design of ZigBee enabled data acquisition system for instantaneous flat-plate collector efficiency calculation. It involves measurement of parameters like inlet and outlet fluid temperatures, ambient temperature and solar radiation intensity. The designed system has a base station and a sensor node. ZigBee wireless communication protocol is used for communication between the base station and the sensor node for wireless data acquisition. The wireless sensor node which is mounted over the collector plate includes the necessary sensors and associated signal-conditioners. An application program has been developed on LabVIEW platform for data acquisition, processing and analysis and is executed in base station PC. Instantaneous flat-plate collector efficiency is computed and reported.
\end{abstract}

Keywords: ZigBee, WSN, flat-plate collector, efficiency calculation, LabVIEW

\section{INTRODUCTION}

$\mathrm{W}$ IRELESS SENSOR network (WSN) offers greater flexibility and mobility in sensor deployment and measurement. Wireless sensors are typically small sized devices used in low cost and low power data acquisition systems. ZigBee technology is a standard protocol stack of IEEE. ZigBee technology has the characteristics suitable for communicating wirelessly with low power consumption for the distance of $100-300 \mathrm{~m}$. ZigBee technology has been successfully deployed in many applications such as water irrigation control [1], street light control [2], temperature monitoring [3], [4], health monitoring [5], environmental parameter monitoring [6]-[8], automobile monitoring [9], etc.

Many research works on the development of "passive" solar energy systems, which involve the integration of several subsystems: flat-plate collectors, heat-storage containers, fluid transport and distribution systems, and control systems, are in progress. The major component unique to passive systems is the flat-plate collector. A typical flat-plate collector is an insulated metal box with a glass or plastic cover called the glazing and a dark-colored absorber plate. This device absorbs the incoming solar radiation, converts it into heat at the absorbing surface, and transfers the heat to a fluid or water flowing through the flatplate collector [10]. The warmed-up fluid carries the heat either directly to the hot water or to a storage subsystem. Flat-plate collectors are the most common solar collectors for solar water-heating systems for home. Measurement of instantaneous flat-plate collector efficiency is important for solar thermal system testing.

At the Regional Solar Testing Centre, Madurai Kamaraj University, Madurai, India, the solar thermal efficiency of different thermal collectors is calculated by acquiring measurement data using wired data loggers. In the conventional method, parameters like inlet and outlet fluid temperatures, ambient temperature and solar radiation are measured manually using appropriate instruments. Inlet and outlet fluid temperatures are measured with Pt-1000 based digital thermometers. The ambient temperature is measured with a thermo-hygrometer. Solar radiation level is measured with a standard pyranometer.

The present system applies a novel wireless approach by applying the ZigBee technology in the measurement of instantaneous flat-plate collector efficiency.

\section{EXPERIMENTAL SETUP}

The ZigBee compatible XBee PRO wireless modules from Digi are used in the present experimental setup for wireless communication and data acquisition. The system consists of a base station and a wireless sensor node. The base station has a PC with an XBee PRO module attached to it through USB port. It serves as a ZigBee coordinator. The wireless sensor node designed for flat-plate collector efficiency measurement consists of four sensors, associated signal conditioners and an XBee PRO module. The block diagram of the experimental setup is shown in Fig.1.

The base station is $100 \mathrm{~m}$ away from the sensor node. The sensor node is accessed from the base station PC using API command set provided by the manufacturer. A LabVIEW program, executed in the base station PC, communicates with wireless sensor node and performs the data acquisition, processing and analysis operations. Fig. 2 shows the flatplate collector with sensor node setup.

The wireless sensor node includes two RTD temperature sensors (Pt-100) to measure inlet and outlet water temperatures of the flat-plate collector, an IC temperature sensor (LM35) to measure ambient temperature and a photodiode (BPW34) to measure intensity of solar radiation. The signal-conditioner for Pt-100 uses a bridge amplifier and produces an output of $9.625 \mathrm{mV}$ for a change of $1^{\circ} \mathrm{C}$ in temperature. LM35 produces output of $10 \mathrm{mV}$ for a change of $1^{\circ} \mathrm{C}$ in temperature. The signal-conditioner for BPW34 uses $\mathrm{I}$ to $\mathrm{V}$ converter to convert the current output of BPW34 to voltage. It produces an output of $580 \mu \mathrm{V}$ for a change of $1 \mathrm{~W} / \mathrm{m}^{2}$ solar radiation intensity. The voltage 
outputs of the signal conditioners are applied to AIN0 AIN3 inputs of XBee PRO module. The XBee PRO module has 4 programmable analog input pins (AIN0-AIN3) and an in-built 10-bit ADC module[11].

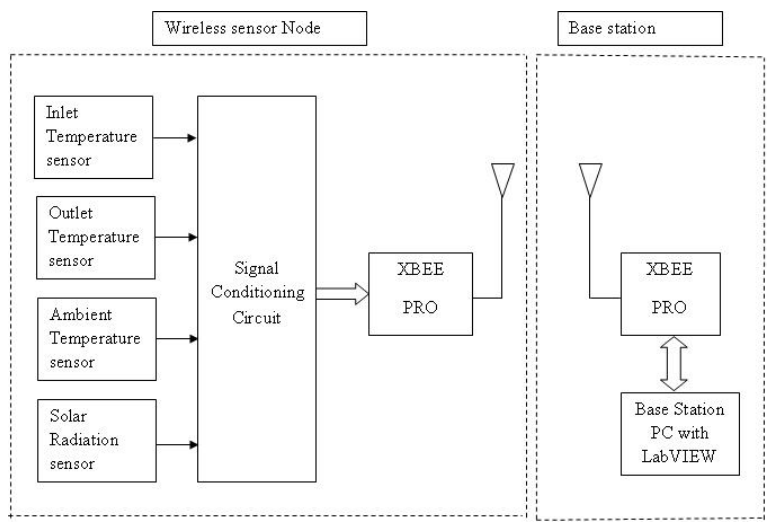

Fig.1. Block diagram of the experimental setup

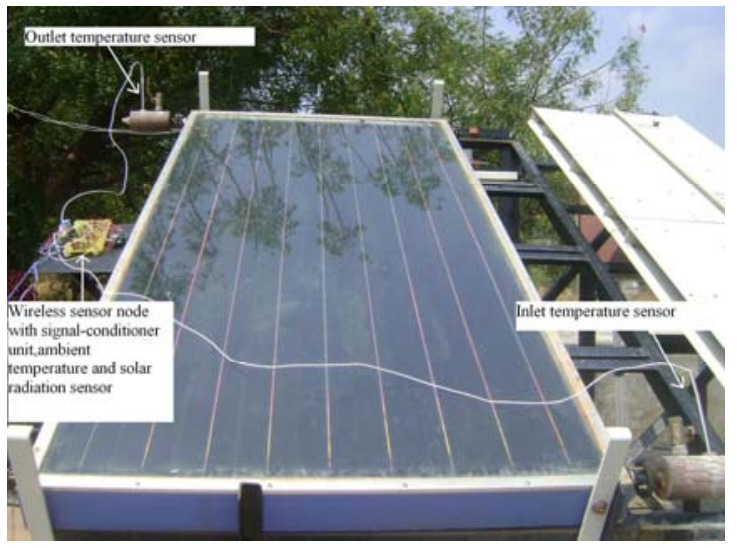

Fig.2. Flat-plate collector with sensor node mounted on it.

\section{SOFTWARE DEVELOPMENT}

A set of API commands provided by Digi has been used to establish the communication between the XBee PRO modules. An application program (VI) has been developed on National Instruments' LabVIEW platform to send the API commands to the wireless sensor node and to get response data. The program utilizes the in-built VISA serial functions, (i) VISA Configure Serial Port.vi, (ii) VISA Write.vi, and (iii) VISA Read.vi to configure and communicate through the virtual COM port. A subVI 'SerWr\&Rd.vi' has been developed to send and receive strings through the virtual COM port. The subVI converts API commands into strings, writes the string of characters to the virtual COM port, waits for 1 second and reads the response data from the virtual COM port.

The application VI performs the following functions:

(i) Configuration of wireless sensor node: The VI selects the wireless sensor node with its 64-bit address and enables the four analog channels, AIN0-AIN3, by sending API commands 'D0' to 'D3' in sequence. The VI sends the 'Apply Changes' (AC) command each time a channel is configured, to reinitialize the wireless sensor node for the changes currently made. Fig.3 shows the configuration operation of the VI.

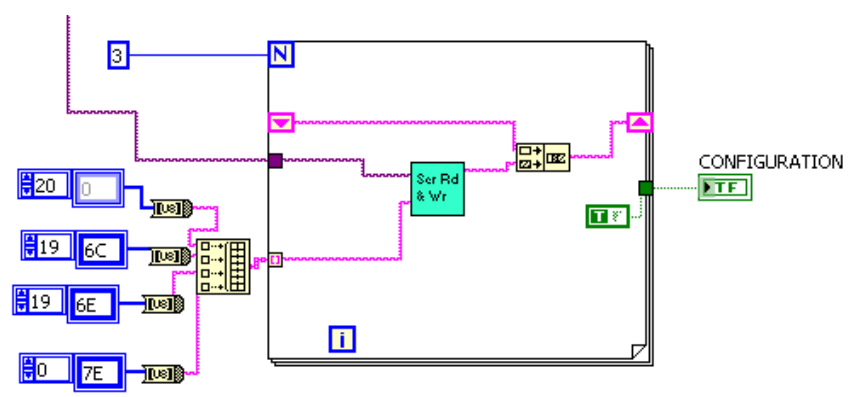

Fig.3. Configuration operation of the VI

(ii) Data acquisition: The VI sends the 'Force Sample' (IS) API command to read all the enabled analog channels. The VI further applies corresponding conversion formula on the received data and computes the measurement parameter values. The VI plots the parameter values on a waveform chart.

(iii) Time stamping: The VI converts the parameter values to a string using in-built function 'array to spreadsheet string.vi' and appends the timestamp to the string by reading the system time using the in-built function 'Get Date/Time String.vi'.

The data acquisition and time stamping operations are repeated at a regular interval of 1 minute for 130 times. Fig. 4 shows the data acquisition and time stamping operations of the VI.

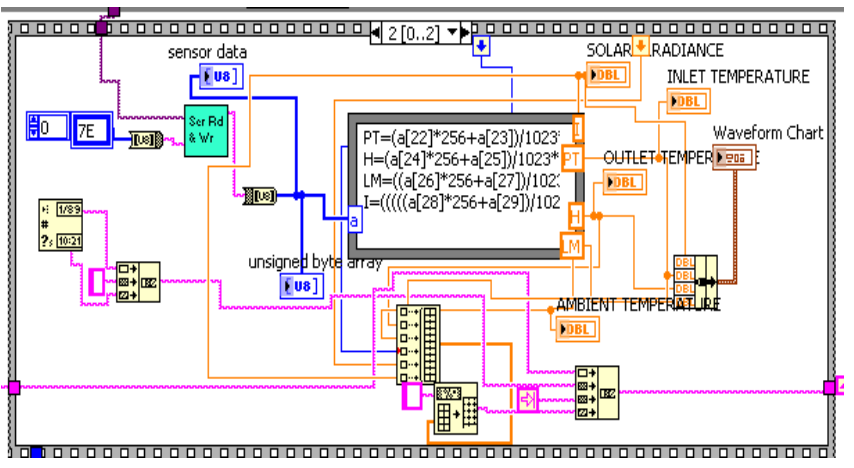

Fig.4. Data acquisition and time stamping operations of the VI.

(iv) File save: The VI writes the appended string to a data file using in-built functions 'open/create/replace file.vi' and 'write to file.vi'. Fig. 5 shows the file save operation of the VI.

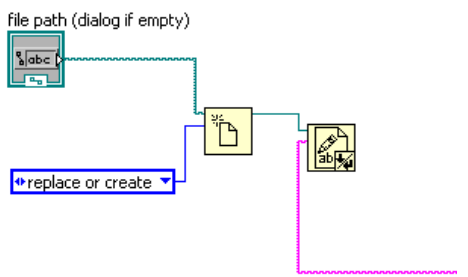

Fig.5. File save operation of the VI 
The front panel of the designed VI displays the measured values as shown in Fig.6.

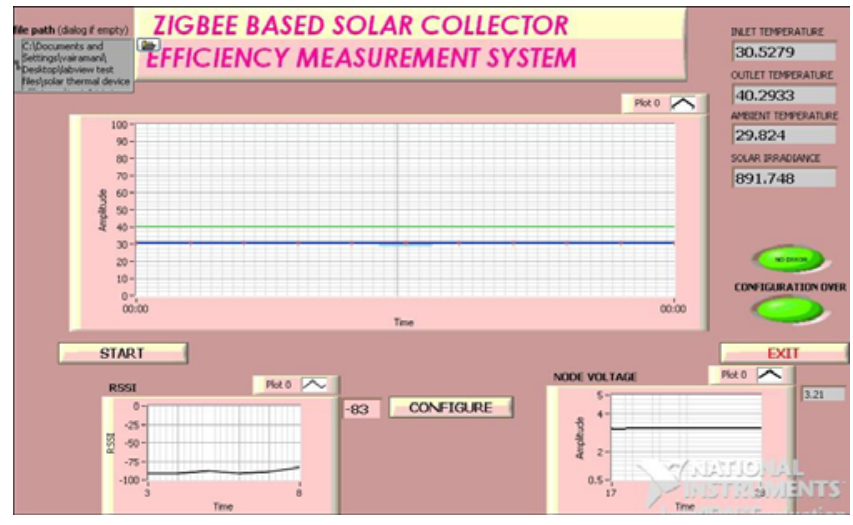

Fig.6. Front-panel of VI.

\section{EFFICIENCY CALCULATION}

Using the data available in the logged file, the instantaneous thermal efficiency is calculated. The instantaneous thermal efficiency of the flat-plate collector is given in equation (1) as [12],

$$
\eta=\frac{m c_{p}\left(T_{f o}-T_{f i}\right)}{A_{c} I_{T}}
$$

Where,

$\eta$ - efficiency of flat plate collector

$m$ - fluid flow rate in $\mathrm{kg} / \mathrm{sec}$

$C p$ - specific heat of water in $\mathrm{kJ} / \mathrm{kg}{ }^{\circ} \mathrm{K}$

$T_{f_{i}}$ - fluid inlet temperature in ${ }^{\circ} \mathrm{C}$

$T_{f 0}$ - fluid outlet temperature in ${ }^{\circ} \mathrm{C}$

$A_{c}$ - area of flat-plate collector in $\mathrm{m}^{2}$

$I_{T^{-}}$- solar radiation incident on the collector plane in $\mathrm{W} / \mathrm{m}^{2}$

\section{RESULTS AND DISCUSSION}

The developed system has been tested with a flat-plate collector in the Regional Solar Testing Centre, Madurai Kamaraj University. The mass flow rate of water was set to $0.03 \mathrm{~kg} / \mathrm{sec}$ and the temperature of inlet water was set to $30^{\circ} \mathrm{C}$. Circulation of water was started at least fifteen minutes before the data acquisition was started to allow the system to reach the steady state. For the calculation of instantaneous flat-plate collector efficiency, two data before solar noon and two data after solar noon were taken. The inlet, outlet, ambient temperature values, solar radiation values and the efficiency values at different times obtained with the present system are given in Table1.

The efficiency of flat-plate collector is the average of four instantaneous efficiency values and is computed as 0.75 . For the same flat-plate collector, the efficiency value has been computed with the data acquired using the conventional data logger at the Regional Solar Testing Centre. The value obtained was 0.73 . It shows that the efficiency value obtained with the present system is well in agreement with the efficiency value obtained with conventional data loggers.
Table 1

Measured values of inlet, outlet and ambient temperatures, solar radiation and calculated values of instantaneous efficiency at different times

\begin{tabular}{|c|c|c|c|c|c|}
\hline Time & $\begin{array}{c}\text { Inlet } \\
{ }^{\circ} \mathrm{C}\end{array}$ & $\begin{array}{c}\text { Outlet } \\
{ }^{\circ} \mathrm{C}\end{array}$ & $\begin{array}{c}\text { Ambient } \\
{ }^{\circ} \mathrm{C}\end{array}$ & $\begin{array}{c}\text { Solar radiation } \\
\mathrm{W} / \mathrm{m}^{2}\end{array}$ & $\begin{array}{c}\text { Instantaneous } \\
\text { Efficiency } \\
\eta\end{array}$ \\
\hline $11: 00$ & 29.9 & 38.35 & 29.2 & 781.7 & 0.677 \\
\hline $11: 30$ & 29.32 & 39.75 & 37.59 & 954.28 & 0.685 \\
\hline $12: 30$ & 29.9 & 40.75 & 39.06 & 765.51 & 0.889 \\
\hline $13: 00$ & 29.9 & 40.89 & 34.6 & 915.38 & 0.752 \\
\hline \multicolumn{7}{|c|}{ Average $\eta$} & 0.75 \\
\hline
\end{tabular}

Fig.7 shows the plot of inlet, outlet water temperatures and ambient temperature over the period of measurement. Fig.8 shows the intensity of solar radiation over the period of measurement.

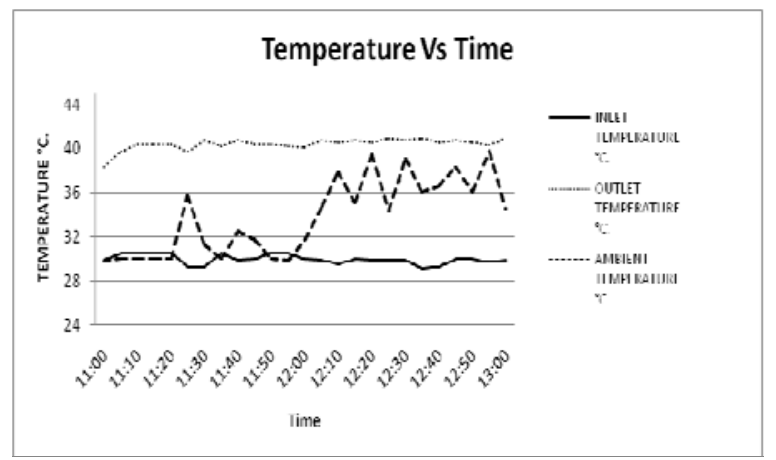

Fig.7. Plot of inlet and outlet water temperatures and ambient temperature vs. time

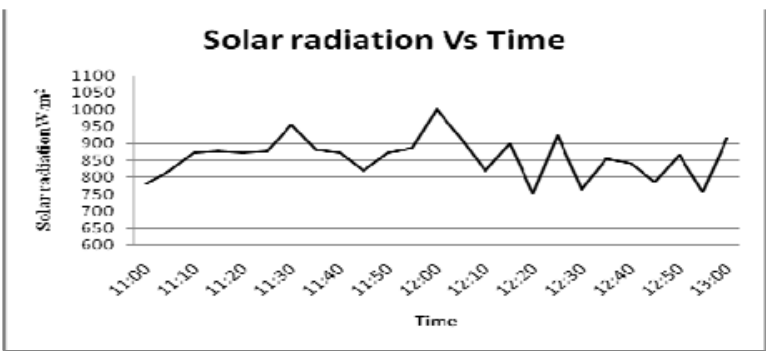

Fig.8. Plot of solar radiation vs. time

\section{CONCLUSIONS}

From the above results, it has been found that the flat plate collector efficiency calculated with data acquired using the present wireless data acquisition system is 0.75 , which matches with the value obtained from the conventional method. The WSN based system is easy to set up, configure and get measurement values. It consumes less power. The WSN based system is better than the conventional measurement method in terms of automatic measurement, cost, reliability, and portability. The developed ZigBee and LabVIEW based data acquisition system eliminates the need for lengthy communication wires and provides networking capabilities. LabVIEW further provides excellent graphical user interface and advanced signal processing functions for research and analysis. 


\section{REFERENCES}

[1] Rasin, Z., Hamzah, H., Aras, M.S.M. (2009). Application and evaluation of high power ZigBee based wireless sensor network in water irrigation control monitoring system. In 2009 IEEE Symposium on Industrial Electronics and Applications (ISIEA 2009), October 4-6, 2009, 548-551.

[2] Lee, J.D., Nam, K.Y., Jeong, S.H., Choi, S.B., Ryoo, H.S., Kim, D.K. (2006). Development of ZigBee based street light control system. In Power Systems Conference and Exposition (PSCE 2006), 2236-2240.

[3] Sehgal, V.K., Nitin Chauhan, D.S., Sharma, R. (2008). Smart wireless temperature data logger using IEEE 802.15.4/ZigBee protocol. In TENCON 2008 - IEEE Region 10 Conference, November 19-21, 2008, 1-6.

[4] Terada, M. (2009). Application of ZigBee sensor network to data acquisition and monitoring. Measurement Science Review, 9 (6), 183-186.

[5] Watthanawisuth, N., Lomas, T., Wisitsoraat, A., Tuantranont, A. (2010). Wireless wearable pulse oximeter for health monitoring using ZigBee wireless sensor network. In ECTI-CON-2010 International Conference, May 19-21, 2010, 575-579.

[6] Wang, Z., Chen, T., Yu, Ch. (2009). ZigBee based environmental temperature monitoring system. In International Symposium on Intelligent Information Systems and Applications (IISA'09), October 28-30, 2009, 285-289.
[7] Valente, A., Morais, R., Serodio, C., Mestre, P., Pinto, S., Cabral, M. (2007). A ZigBee sensor element for distributed monitoring of soil parameters in environmental monitoring. In IEEE SENSORS 2007 Conference, October 28-31, 2007, 135-138.

[8] Santos, F.E.S., Azevedo, J.A.R. (2009). Application of ZigBee and Bluetooth to urban ambient monitoring and guidance. In 8th International Conference and Workshop on Ambient Intelligence and Embedded Systems, September 2009, 187-190.

[9] Tavares, J., Velez, F.J., Ferro, J.M. (2008). Application of wireless sensor networks to automobiles. Measurement Science Review, 8 (3), 65-70.

[10] Rhushi Prasad, P., Byregowda, H.V., Gangavati, P.B. (2010). Experiment analysis of flat plate collector and comparison of performance with tracking collector. European Journal of Scientific Research, 40 (1), 144-155.

[11] Digi International Inc. (2008). ZNet2.5/XBee-PRO OEM RF Modules.

[12] Sukhatme, S.P. (2008). Solar Energy: Principles of Thermal Collection and Storage, $2^{\text {nd }}$ edition. Tata Mc-Graw-Hill Publishing Company, 99-166.

Received March 11, 2011. Accepted May 30, 2011. 